\section{(1)}

CrossMark

\title{
Variation in lung function as a marker of adherence to oral and inhaled medication in cystic fibrosis
}

\author{
Helen White ${ }^{1,2}$, Nicola Shaw ${ }^{2}$, Sarah Denman², Kim Pollard ${ }^{2}$, Sarah Wynne $^{2}$ and \\ Daniel Gavin Peckham ${ }^{2,3}$
}

Affiliations: ${ }^{1}$ Nutrition and Dietetic Group, School of Clinical and Applied Science, Leeds Beckett University, Leeds, UK. ${ }^{2}$ Adult Cystic Fibrosis Unit, Leeds Teaching Hospitals NHS Trust, Leeds, UK. ${ }^{3}$ Leeds Institute of Biomedical and Clinical Sciences, Faculty of Medicine and Health, University of Leeds, Leeds, UK.

Correspondence: H. White, Nutrition and Dietetic Group, School of Clinical and Applied Science, Leeds Beckett University, Leeds, UK. E-mail: H.Whitealeedsbeckett.ac.uk

@ERSpublications

The coefficient of variation of FEV1 is a significant objective predictor of adherence in cystic fibrosis http://ow.ly/kcrb307S2T3

Cite this article as: White $\mathrm{H}$, Shaw N, Denman S, et al. Variation in lung function as a marker of adherence to oral and inhaled medication in cystic fibrosis. Eur Respir J 2017; 49: 1600987 [https://doi.org/ $10.1183 / 13993003.00987-2016]$.

ABSTRACT The aim of this study was to characterise adherence in an adult population with cystic fibrosis (CF) and to investigate if variation in lung function was a predictor of adherence to treatment.

The adherence of patients aged $\geqslant 16$ years from an adult CF centre was measured by medication possession ratio (MPR) and self-report. Patients were assigned to one of three adherence categories $(<50 \%$, 50 to $<80 \%, \geqslant 80 \%$ ) by their composite score (MPR). Ordinal regression was used to identify predictors of adherence, including coefficient variation measures for forced expiratory volume in $1 \mathrm{~s}$ (FEV1), weight and C-reactive protein concentration, measured from 6 months and 12 months before baseline.

MPR data for 106 of 249 patients (mean age 29.8 \pm 9.2 years) was retrieved, indicating a mean adherence of $63 \%$. The coefficient of variation for $\mathrm{FEV}_{1}$ was inversely related to adherence and was a univariate predictor of adherence ( 6 months: OR $0.92,95 \%$ CI $0.87-0.98, \mathrm{p}=0.005 ; 12$ months: OR 0.94, 95\% CI $0.93-0.99, \mathrm{p}=0.03$ ) and remained significant in the final models. The coefficient of variation of weight and $\mathrm{C}$-reactive protein were not predictive of adherence.

The coefficient of variation of FEV1 was identified as an objective predictor of adherence. Further evaluation of this potential marker of adherence is now required.

This article has supplementary material available from erj.ersjournals.com

Received: May 172016 | Accepted after revision: Dec 052016

Support statement: This work was supported by Gilead Science (grant no. IN-UK-205-D001) and the UK Clinical Research Network (ID 13624). Funding information for this article has been deposited with the Crossref Funder Registry.

Conflict of interest: Disclosures can be found alongside this article at erj.ersjournals.com. 


\section{Introduction}

Advances in early diagnosis and treatment of cystic fibrosis (CF) have resulted in significant improvements in survival, with many patients living into adulthood and middle age $[1,2]$. This success has been achieved through specialised multidisciplinary care in combination with intense treatment regimens which are time-consuming and negatively impact on daily life. Poor adherence to treatment remains a significant problem, being as low as 40-50\% [3-7]. Adherence problems can negatively influence health outcomes such as pulmonary exacerbations [8], health-related quality of life [3] and healthcare costs [6-9]; these trends are also apparent across other respiratory diseases [10-12].

Consensus regarding the accurate measurement of adherence is lacking and has proved difficult in day-to-day practice. Subjective self-report with objective measures that include pharmacy collection, medication possession ratio (MPR) and chipped hardware (such as the i-neb [4]) are frequently used as part of good practice in reporting study findings. Whilst all of these have inherent limitations [7], objective physiological measures that could more accurately define adherence are lacking.

Studies in other chronic diseases have recently highlighted the association between variation in physiological measures, such as blood pressure [13-15] and immunosuppressive therapy [16], and adherence to medication. These objective physiological measures may help to characterise poor adherence and trigger interventions to support better outcomes. Attention has focused on the variability of lung function as an improved predictor of lung decline in CF, above that of FEV1 alone. The hypothesis is that the variability of this measure is a more sensitive marker that can reflect exacerbations, individual pulmonary variation and adherence [17].

In $\mathrm{CF}$ there is evidence that low rates of medication adherence are associated with increased pulmonary exacerbations $[8,18]$. Given the maintenance effect of medications such as nebuliser therapies on respiratory function, it is therefore plausible that poor adherence could be associated with greater variation in lung function. A variability measure that accounts for natural changes, exacerbations and individual variation over time could also provide greater predictive accuracy than shown in studies utilising an association between adherence and the single measure of baseline FEV1 [8].

In the day-to-day management of patients with CF, we have recognised apparent differences in the fluctuation of lung function, weight and infection markers in patients known to have poor adherence. Patterns in diseases such as these have only become apparent after the implementation of disease-specific electronic patient records containing rich clinical "real-time" diagnostic, biochemical, anthropometric, pulmonary function and pathology data [19]. These detailed longitudinal data, which are routinely displayed graphically, have provided a basis for testing whether variation indices could predict adherence within a population with CF.

The aim of this study was to characterise adherence in a large adult population with CF and to test the hypothesis that increased variation in lung function is an indicator of poor adherence to treatment.

\section{Methods}

Participants

Patients attending a large regional adult CF unit were recruited from December 2012 to August 2013. Participants were identified from an electronic register of 400 patients, were $\geqslant 16$ years, and had a diagnosis of $\mathrm{CF}$ as defined by the presence of a positive clinical phenotype with either two CF-causing mutations and/or two positive sweat tests. Participants were consecutively invited to participate in the study when they attended a routine outpatient visit at a time of clinical stability. Patients attended every 2-3 months, in line with local policy. Exclusion criteria included pregnancy, neurological disease, malignancy and renal disease to avoid potential influences on adherence patterns.

\section{Measures}

An experienced physiotherapist had recorded forced expiratory volume in $1 \mathrm{~s}$ (FEV1) and forced vital capacity (FVC) over the preceding 12 months using a calibrated, compact spirometer (Vitalograph, Buckingham, UK). The best value from a series of at least three attempts was used, in accordance with guidelines [20]. In addition, at baseline we recorded age; sex; genotype; weight; height; body mass index (BMI); concentrations of vitamins $\mathrm{A}, \mathrm{D}$ and $\mathrm{E}$ and C-reactive protein (CRP); and the number of days treated intravenously with antibiotics within the last year.

The coefficient of variation $(\mathrm{CoV})$ for $\mathrm{FEV} 1$ was calculated by extracting all consecutive highest then lowest values for FEV1 for 6 and 12 months prior to baseline from the electronic health record (EHR). These timescales were chosen to align with data on CoV FEV1 retrieved from pharmacy records (6 months) and to examine the impact of longer-term patterns of variation of CoV FEV1 on adherence (12 months). The total, mean and standard deviation of all values for FEV1 for each participant were calculated. The equation for $\mathrm{CoV}_{\mathrm{FEV}} 1$ was then calculated using the standard deviation of FEV1 divided by the mean FEV1. The CoV for weight and CRP were similarly examined. 
Adherence measures

Pharmacy collection

Individual medications and prescribed days of treatment were retrieved from the EHR for the time period $\leqslant 6$ months prior to the baseline index date, a timescale chosen to enable greater comparability with the level of self-reported adherence reported by the Disease Management Interview-Cystic Fibrosis (DMI-CF) disease-specific questionnaire [7]. Community pharmacy details were abstracted from the EHR and participants gave contact details for all other community pharmacies used within this time period. A letter of request was sent to all pharmacies together with a copy of the patient's signed consent form requesting the amount of medication prescribed for $\leqslant 6$ months prior to the baseline index date. Up to two repeat requests were made by telephone to maximise prescription data return. Similarly for patients using the i-neb, the prescribed number of doses was retrieved from the EHR and medication uptake then downloaded from the i-neb for $\leqslant 6$ months prior to the baseline index date. The patient's MPR was then adjusted for all medications prescribed during inpatient admission and any outpatient prescriptions issued from the ward. The MPR was calculated from at least two prescription collections for each individual medication and then averaged to obtain a composite MPR. MPR was calculated as medication dispensed divided by the number of days medication prescribed, multiplied by 100 .

\section{Adherence self-report}

For each participant, a record of all routinely prescribed medications was generated at baseline from the EHR. All subjects completed a DMI-CF self-report adherence questionnaire at baseline [7]. Percentage adherence to each prescribed medication was then calculated by dividing the reported dose by the prescribed dose for the each of the following: airway clearance, recombinant DNase, bronchodilators, inhaled steroids, pancreatic enzyme replacement therapy, nutrition, oral nutritional supplements or nasogastric/gastrostomy feed, vitamins, oral antibiotics, inhaled antibiotics and insulin.

A composite adherence measure was calculated, omitting self-reported adherence to airway clearance, nutrition and insulin, to enable a composite measure comparable to adherence by MPR. Composite adherence was calculated as the sum of all medications patients were prescribed on the EHR, divided by the total number of self-reported medications taken.

\section{Statistical analysis}

Patient characteristics across medication and treatment adherence (MPR) were compared using a Pearson's Chi-squared test (categorical variables) or ANOVA (continuous variables). The Wilcoxon sign rank test was used to compare self-reported with pharmacy record-obtained adherence.

Patients were assigned to one of three categories according to the MPR-reported adherence $(<50 \%$ adherence, 50 to $<80 \%$ adherence, and $\geqslant 80 \%$ adherence), a classification used in previous research in CF [8]. Ordinal regression was used to identify univariate predictors of adherence for testing in a multivariate model, using CoV measures 6 and 12 months preceding baseline, respectively. Patients' genotypes were classified into three categories (delta F508 homozygous, delta F508 heterozygous and "other"). Only univariate variables with a $\mathrm{p}$-value of $<0.1$ were used in the multivariate model. A forwards and backwards stepwise process was used to construct a final model.

\section{Model evaluation}

A Chi-squared score test was used to evaluate the proportional odds assumption and whether this was violated. We also calculated single score tests for each covariate and included the parallel lines assumption to check this assumption. We tested for multicollinearity by calculating the variance inflation factor for each predictor. We examined the likelihood ratio, score and Wald tests to determine the improvement of the multiple linear regression model over the intercept model.

All analyses was implemented using SPSS 22 (SPSS Inc., Chicago, IL, USA). p-values $<0.05$ were considered significant.

\section{Ethical approval}

The study received ethical approval from London City and East ethics committee (ref: 12/LO/1776) and met all applicable institutional regulations at Leeds Teaching Hospitals NHS Trust, UK.

\section{Results}

\section{Participant characteristics}

Of 267 patients invited to participate in the study, 250 subjects were enrolled; 17 declined to participate, with 15 stating that they did not want to participate in this specific study and two stating that they did not 
wish to participate in studies in general. A further participant withdrew prior to analysis, leaving 249 participants. Pharmacy refill data were obtained for 106 of these patients.

With the exception of the presence of diabetes, no differences were observed in characteristics between those with pharmacy refill data and those without (table 1). Adherence by MPR was subsequently used in further analyses.

Baseline characteristics are shown in table 2. Participants were predominantly homozygous for the delta F508 mutation and had good nutritional status. Clinical and demographic characteristics were similar between adherence categories (MPR) (table 2), but were significantly different for age, number of medications, weight, BMI and levels of vitamins $\mathrm{A}, \mathrm{D}$ and $\mathrm{E}$.

\section{Adherence measures}

Pharmacy refill collection was retrieved for 106 of the 249 participants and 249 participants completed the DMI-CF self-report adherence questionnaire. Comparison of the two adherence measures revealed significant differences for rDNase, inhaled steroids, oral antibiotics, pancreatic enzyme supplementation, nutritional supplements and vitamins. MPR-reported adherence was consistently below that of self-reported adherence for all medications and treatments (figure 1). For three aspects of adherence an accurate MPR could not be calculated, either owing to lack of verifiable data (nutrition and airway clearance) or highly variable dosage (insulin). The composite MPR score for adherence was $63.4 \pm 25.3 \%$ and for self-report was $79.9 \pm 19.7 \%$ (figure 1 ), with only $32.1 \%$ of subjects lying within the highest adherence category (table 2 ). A score of zero was recorded for MPR for three patients: a comparable score of 3\% was obtained for self-reported adherence for one patient whereas the remaining two patients had no further adjustment to MPR after checking for inpatient admission and prescriptions issued at end of inpatient stay.

\section{Variation measures}

The mean $\mathrm{CoV}$ for $\mathrm{FEV} 1$, calculated from the preceding 6-month period, was $11.4 \pm 7.4 \%$ and the CoVs for weight and CRP were $2.1 \pm 1.4 \%$ and $35.8 \pm 41.0 \%$, respectively. Patients with poor adherence $(<50 \%)$ had on average a $4.67 \%$ higher CoV FEV1 and a $7.3 \%$ higher CoV CRP compared with those with good adherence $(>80 \%)$. The $\mathrm{CoV}$ for weight remained stable across MPR adherence categories (figure 2). Values were similar using data from the preceding 12-month period, with a CoV FEV1 of $11.4 \pm 7.2 \%$, a CoV weight of

\begin{tabular}{|c|c|c|c|}
\hline Characteristic & MPR data available & No MPR data available & $\mathrm{p}$-value \\
\hline Subjects $\mathrm{n}$ & 106 & 143 & \\
\hline Age years & $29.8 \pm 8.2$ & $29.5 \pm 9.8$ & 0.8 \\
\hline Male & $46(43.4)$ & 57 (39.9) & 0.6 \\
\hline \multicolumn{4}{|l|}{ Genotype } \\
\hline F508/F508 & 72 (68.0) & $81(56.7)$ & 0.1 \\
\hline F508/heterozygous & $31(29.2)$ & $1(35.6)$ & \\
\hline Other & $3(2.8)$ & $11(7.7)$ & \\
\hline Cystic fibrosis related diabetes & $17(16)$ & $11(7.7)$ & 0.04 \\
\hline \multicolumn{4}{|l|}{ Microbiological status } \\
\hline Non-Pseudomonas & $23(21.7)$ & $32(22.4)$ & 0.2 \\
\hline Pseudomonas & $75(70.8)$ & $88(61.5)$ & \\
\hline Burkholderia cepacia & $5(4.7)$ & $12(8.4)$ & \\
\hline Mycobacterium abcessus & $3(2.8)$ & $11(7.7)$ & \\
\hline Weight kg & $64.0 \pm 13.6$ & $64.3 \pm 13.7$ & 0.9 \\
\hline BMI $\mathrm{kg} \cdot \mathrm{m}^{-2}$ & $22.4 \pm 3.6$ & $22.6 \pm 4.0$ & 0.6 \\
\hline FEV $1 \%$ predicted & $60.2 \pm 24.5$ & $62.4 \pm 25.9$ & 0.5 \\
\hline FVC $\%$ predicted & $78.7 \pm 22.1$ & $80.2 \pm 24.0$ & 0.6 \\
\hline $\mathrm{HbA} 1 \mathrm{c}$ mmol-mol ${ }^{-1}$ & $45.2 \pm 13.8$ & $44.5 \pm 12.1$ & 0.7 \\
\hline $\begin{array}{l}\text { Days treated with i.v. antibiotics } \\
\text { (preceding } 12 \text { months) }\end{array}$ & $29.1 \pm 40.0$ & $28.0 \pm 39.4$ & 0.8 \\
\hline Number of medications & $13.8 \pm 4.5$ & $13.1 \pm 4.7$ & 0.3 \\
\hline
\end{tabular}

Data are presented as mean \pm SD or $\mathrm{n}(\%)$ unless otherwise stated. A two-sample t-test was used for normally distributed variables and Pearson's Chi-squared test for categorical variables. MPR: medication possession ratio; BMI: body mass index; FEV1: forced expiratory volume in $1 \mathrm{~s}$; FVC: forced vital capacity; HbA1c: haemoglobin A1c; i.v.: intravenous. 
TABLE 2 Participant characteristics according to adherence measured by medication possession ratio

\begin{tabular}{|c|c|c|c|c|c|}
\hline \multirow[t]{2}{*}{ Characteristic } & \multirow[t]{2}{*}{ Total study population } & \multicolumn{3}{|c|}{ Adherence categories } & \multirow[b]{2}{*}{ p-value } \\
\hline & & Poor $\#$ & Moderate & Good $^{+}$ & \\
\hline Subjects & $106(100)$ & $34(32.1)$ & $38(35.8)$ & $34(32.1)$ & 0.86 \\
\hline Sex $(M / F)$ & $60 / 46$ & $17 / 17$ & $23 / 15$ & $20 / 14$ & 0.63 \\
\hline Genotype & & & & & 0.60 \\
\hline F508/F508 & 72 (67.9) & $24(22.6)$ & $23(21.7)$ & $25(23.5)$ & \\
\hline Cystic fibrosis related diabetes & $17(16)$ & $2(1.9)$ & $10(9.4)$ & $5(4.7)$ & 0.06 \\
\hline Microbiological status & & & & & 0.3 \\
\hline Non-Pseudomonas & 23 (21.7) & $7(6.6)$ & 9 (8.5) & $7(6.6)$ & \\
\hline Pseudomonas & $75(70.8)$ & $25(23.6)$ & $24(22.6)$ & $26(24.5)$ & \\
\hline Burkholderia cepacia & 5 (4.7) & 0 & $4(3.8)$ & $1(0.9)$ & \\
\hline Mycobacterium abcessus & $3(2.8)$ & 2 (1.9) & $1(0.9)$ & 0 & \\
\hline BMI $\mathrm{kg} \cdot \mathrm{m}^{-2}$ & $22.4 \pm 3.6$ & $20.8 \pm 2.8$ & $22.9 \pm 3.1$ & $23.4 \pm 4.3$ & 0.005 \\
\hline FEV $1 \%$ predicted & $60.1 \pm 24.6$ & $62.7 \pm 24.2$ & $58.0 \pm 25.8$ & $60.3 \pm 24.0$ & 0.71 \\
\hline FVC $\%$ predicted & $78.6 \pm 22.2$ & $79.3 \pm 23.1$ & $75.8 \pm 21.9$ & $81.5 \pm 21.7$ & 0.55 \\
\hline $\mathrm{HbA} 1 \mathrm{c} \mathrm{mmol} \cdot \mathrm{mol}^{-1}$ & $45.2 \pm 13.8$ & $41.7 \pm 10.7$ & $49.1 \pm 18.3$ & $44.3 \pm 8.9$ & 0.07 \\
\hline Vitamin A $\mu \mathrm{mol} \cdot \mathrm{L}^{-1}$ & $1.7 \pm 0.8$ & $1.4 \pm 0.60$ & $1.9 \pm 1.0$ & $1.8 \pm 0.53$ & 0.02 \\
\hline Vitamin $\mathrm{D} \mathrm{mmol} \cdot \mathrm{L}^{-1}$ & $66.6 \pm 26.0$ & $51.8 \pm 23.3$ & $72.9 \pm 26.3$ & $74.0 \pm 22.7$ & 0.001 \\
\hline Vitamin $\mathrm{E} \mathrm{mg} \cdot \mathrm{L}^{-1}$ & $28.2 \pm 10.7$ & $22.4 \pm 10.1$ & $29.9 \pm 9.6$ & $31.7 \pm 10.6$ & $<0.001$ \\
\hline
\end{tabular}

Data are presented as mean \pm SD or $\mathrm{n}(\%)$ unless otherwise stated. ANOVA was used for normally distributed variables and Pearson's Chi-squared test for categorical variables. M: male; F: female; BMI: body mass index; FEV1: forced expiratory volume in $1 \mathrm{~s}$; FVC: forced vital capacity; HbA1c: haemoglobin A1c; i.v.: intravenous. ": $<50 \%$; ๆ : 50 to $<80 \%{ }^{+}: \geqslant 80 \%$; ${ }^{\S}$ : difference across categories.

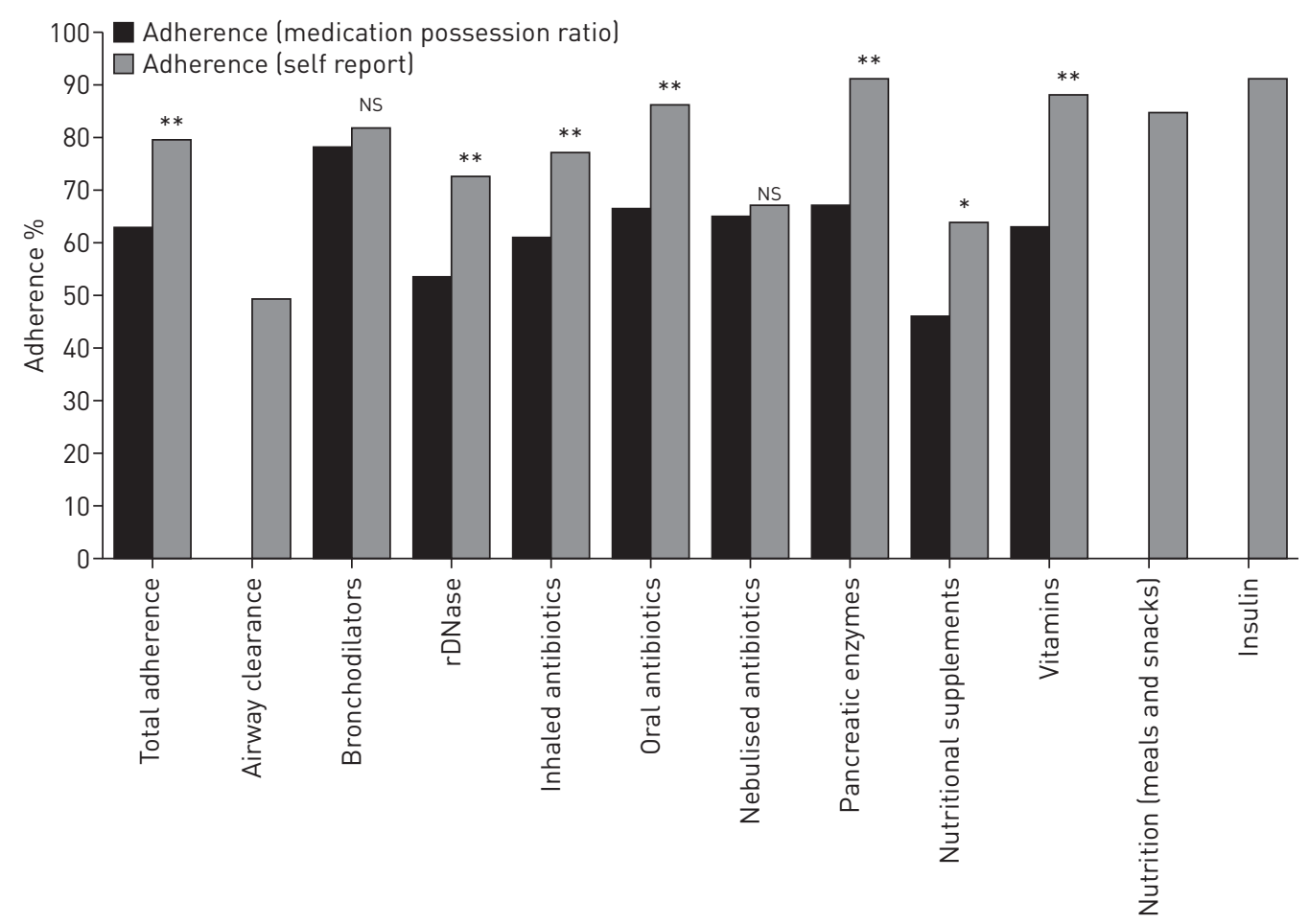

FIGURE 1 Adherence measured by medication possession ratio (MPR) and self-report. Composite self-reported adherence calculated only from medications and treatments with a corresponding MPR value. *: $p<0.05 ;{ }^{* *}: p<0.01$; NS: nonsignificant. 
$2.9 \pm 2.1 \%$ and a CoV CRP of $46.7 \pm 46.4 \%$. In total, 38 out of 106 participants with MPR data (35.8\%) had a stable CRP concentration of $<10 \mathrm{mg} \cdot \mathrm{L}^{-1}$ throughout the period of the study, with zero fluctuation.

\section{Univariate and multivariate analysis}

In an unadjusted ordinal model, predictors of adherence (MPR) were age, number of medications prescribed, BMI, CoV FEV1 (6 months preceding), and vitamins D and E (table 3). Following a stepwise regression, CoV FEV1, number of medications and BMI remained significant (figure 3). The odds of being in a higher adherence category increased with every unit of BMI, each 1\% reduction in CoV FEV1 and each additional medication (figure 3). This was replicated using CoV FEV1 (12 months preceding).

\section{Discussion}

This is the first study to demonstrate a significant inverse relationship between CoV FEV 1 and adherence to treatment in adults with CF. This was true of values based on lung function measures taken up to 6 months and 12 months preceding baseline. Similar inverse relationships in physiological parameters have recently been reported in other chronic diseases. For example, the $\mathrm{CoV}$ of blood pressure, renal function and peak flow increased with reduced adherence to antihypertensive, immunosuppressant and asthma therapy, respectively $[13-16,21]$. These observations suggest that variability in physiological parameters could be an important marker of adherence to treatment in chronic disease more generally.

The underlying mechanism for the predictive value of CoV FEV1 was not addressed in the current study but could reflect poor disease control and reduced adherence to physiotherapy and nebulised therapy. Delays in patients seeking medical intervention at times of exacerbation could also be important, because it is not infrequent for patients with poor adherence to have dramatically improved lung function during hospital admission, resulting in larger variation in lung function than is seen in those who enter treatment at an earlier stage.

It can be argued that in patients with asthma, in whom lung function is routinely used as an objective measure of airway obstruction, the disease is also characterised by a high degree of variability, demonstrated by peak flow and FEV1 falling abruptly on treatment withdrawal. In contrast, CF is associated with endobronchial infection, mucus retention and, to a lesser extent, airway hypersensitivity. The withdrawal of inhaled corticosteroids in patients with CF without asthma appears to have no impact on changes in lung function or use of antibiotics and rescue bronchodilators [22]. This suggests that the variation in lung function in this study is not explained by the diurnal variation or hyper-responsiveness reported in asthma.

Weight loss can occur as a result of reduced adherence to pancreatic and oral supplements and increased energy expenditure during episodes of pulmonary infection. Surprisingly, CoV weight was consistent in all groups, with high levels of reported adherence to nutritional recommendations and pancreatic enzyme replacement therapy in patients with a stable BMI in the mid-normal range $\left(22.5-22.8 \mathrm{~kg} \cdot \mathrm{m}^{-2}\right)$. These findings, and the inclusion of BMI as a positive predictor of adherence in the final model, could reflect better patient engagement with nutritional recommendations. Similarly, the inflammatory marker CRP was not a predictor of adherence; this could in part have been due to normal CRP concentrations in many of the patients and routine early antibiotic intervention prior to elevation in CRP.

Age was a significant positive predictor of MPR adherence in the univariate but not the final model. This confirms the complexity of adherence measurement and the difficulties in defining a single marker alone. Whilst reduced adherence is well documented during the transition from childhood into adolescence and

FIGURE 2 Relationship between coefficient variation measures and adherence, measured by medication possession ratio. CoV: coefficient of variation;

$\mathrm{FEV} 1$ : forced expiratory volume in $1 \mathrm{~s}$; CRP: C-reactive protein.

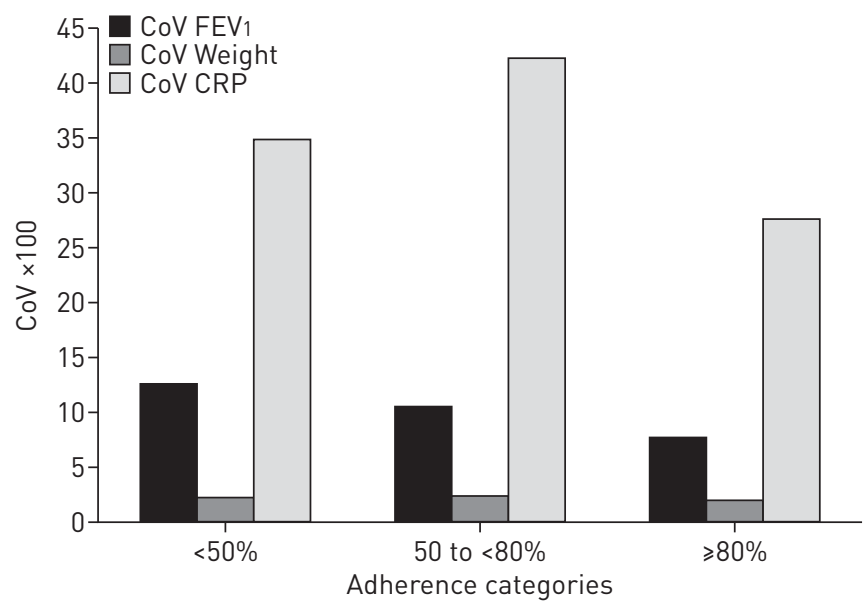


TABLE 3 Univariate analysis of predictors of adherence measured by medication possession ratio

\begin{tabular}{|c|c|c|}
\hline \multirow[t]{2}{*}{ Characteristic } & \multicolumn{2}{|c|}{ Adherence (MPR) } \\
\hline & p-value & OR $(95 \% \mathrm{CI})$ \\
\hline Age years & 0.03 & $1.05(1.01-1.11)$ \\
\hline Sex & 0.46 & $1.31(0.64-2.65)$ \\
\hline \multicolumn{3}{|l|}{ Genotype } \\
\hline Delta F508 homozygous & 0.55 & $1.93(0.22-16.81)$ \\
\hline Delta F508 heterozygous & 0.58 & $1.87(0.20-17.87)$ \\
\hline Other & & (Reference) \\
\hline Number of medications & 0.008 & $1.12(1.03-1.22)$ \\
\hline $\begin{array}{l}\text { Days treated with i.v. antibiotics } \\
\text { (preceding } 12 \text { months) }\end{array}$ & 0.61 & $1.00(0.99-1.01)$ \\
\hline BMI $\mathrm{kg} \cdot \mathrm{m}^{-2}$ & 0.004 & $1.18(1.05-1.32)$ \\
\hline FEV $1 \%$ predicted & 0.68 & $1.0(0.98-1.01)$ \\
\hline HbA1c & 0.55 & $1.01(0.98-1.03)$ \\
\hline \multicolumn{3}{|l|}{6 months preceding } \\
\hline CoV FEV 1 & 0.005 & $0.92(0.87-0.98)$ \\
\hline CoV weight & 0.60 & $0.94(0.73-1.20)$ \\
\hline CoV CRP & 0.80 & $1.00(0.99-1.01)$ \\
\hline \multicolumn{3}{|l|}{12 months preceding } \\
\hline CoV FEV 1 & 0.02 & $0.94(0.89-0.99)$ \\
\hline CoV weight & 0.29 & $0.92(0.79-1.07)$ \\
\hline CoV CRP & 0.14 & $1.00(0.99-1.00)$ \\
\hline Vitamin A & 0.09 & $1.57(0.93-2.65)$ \\
\hline Vitamin D & 0.001 & $1.02(1.01-1.04)$ \\
\hline Vitamin E & $<0.001$ & $1.07(1.03-1.11)$ \\
\hline FEV1 decline $\%$ & 0.91 & $1.00(0.98-1.03)$ \\
\hline Cystic fibrosis related diabetes & 0.40 & $\begin{array}{l}0.65 \text { (0.25-1.70) } \\
\text { (Reference) }\end{array}$ \\
\hline \multicolumn{3}{|l|}{ Microbiological status } \\
\hline Non-Pseudomonas & 0.22 & $4.9(0.38-62.5)$ \\
\hline Pseudomonas & 0.20 & $5.1(0.43-59.7)$ \\
\hline Burkholderia cepacia & 0.19 & $6.9(0.37-129.5)$ \\
\hline Mycobacterium abcessus & & (Reference) \\
\hline
\end{tabular}

Test of parallel lines nonsignificant for all variables (MPR). MPR: medication possession ratio; i.v.: intravenous; $\mathrm{BMI}$ : body mass index; FEV1: forced expiratory volume in $1 \mathrm{~s}$ : $\mathrm{HbA1c}$ : haemoglobin A1c; CoV: coefficient of variation; CRP: C-reactive protein.

young adulthood [5, 9, 23], the impact of age as adulthood progresses is less clear. QuiTTNER et al. examined MPR in 3827 patients and showed a decline in adherence that stabilised in the fourth decade of
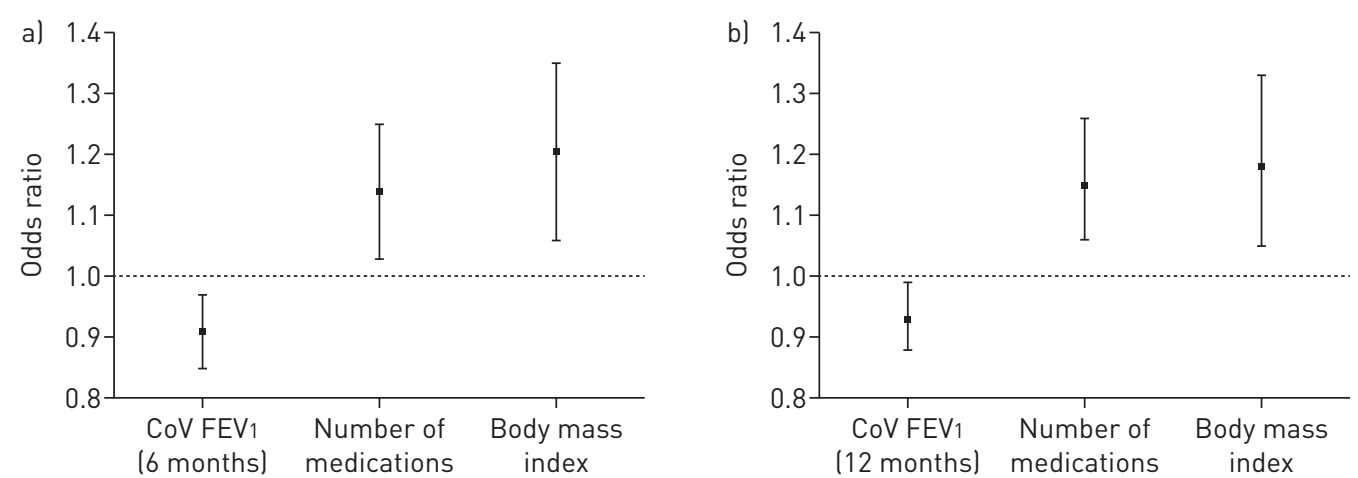

FIGURE 3 Final models of inverse and positive predictors of adherence by medication possession ratio (MPR) using the coefficient of variation of the forced expiratory volume in $1 \mathrm{~s}$ (CoV FEV1). a) Final model using data from preceding 6 months. Score test for the proportional odds assumption: Chi-squared=13.6, $p=0.06$. Goodness of fit test of overall model (likelihood ratio): Chi-squared(7)=28.6, $p<0.0001$. Pseudo R2 (Nagelkerke) $=27.4$. b) Final model using data from preceding 12 months. Score test for the proportional odds assumption: Chi-squared=10.2, $p=0.18$. Goodness of fit test of overall model (likelihood ratio): Chi-squared(7)=25.1, $p<0.0001$. Pseudo R2 (Nagelkerke)=27.1. Both models were adjusted for the presence of cystic fibrosis related diabetes and microbiological status. 
life, a trend replicated by others [9]. Conversely, studies of smaller sample sizes have indicated a positive impact of age [24] whilst acknowledging the wide variation between individuals. Our own results indicate that adherence improves with age, suggesting that interventions to improve adherence should be targeted towards those in early adulthood.

A significant predictor of adherence in the overall models was the number of medications prescribed. This relationship was replicated when using self-reported adherence (supplementary table S1). As adherence category improved, patient medications rose from 10 to 14 medications daily. This positive association contrasts with previous reports that associate treatment burden with barriers to adherence [25, 26], but agrees with findings by QUITTNER et al., who reported a positive association between medication regimen complexity and adherence [9]. However, their proposal that a more complex regimen could be a proxy for disease severity and a greater willingness to undertake prescribed medication was not borne out in our own study. Whilst an upward trend in disease severity was observed, this was not significant, nor was there a difference in the number of days treated intravenously with antibiotics across adherence categories. Other authors have also shown that high treatment burden is independent of disease severity [27]. Why increased medication was associated with improved adherence in our study is therefore unclear. We hypothesise that increased treatment burden could reflect improved clinical control, especially as it is our practice to stop or change medications in partnership with patients in response to poor treatment uptake. It is not infrequent that the team stop all treatment in patents with very poor adherence in order to start again, adjusting therapy accordingly. Another possibility is that some individuals are "resilient" and have developed specific coping strategies that enable them to perform this complex regimen on a regular basis.

Adherence measured by MPR was consistently below that measured by self-report with a mean discrepancy of $14 \%$ for the composite scores. Similar trends are consistent in the literature, although there is little consensus as to how it might best be addressed in reporting data. QuiTTNER et al. have advocated triangulation of data with at least two measures employed, integrated through regression analysis into a single index [7]. We chose to report adherence by MPR and self-reported measures, noting that neither provide a definitive measure of adherence. The discrepancies observed agree with previous reports in adult cohorts, physiotherapy being the least frequently adhered-to treatment (49\%) and pancreatic enzyme replacement therapy the best (91\%) [28]. In general, adherence to respiratory treatments was poorer than to nutritional therapies, suggesting that challenges in improving treatment uptake have changed little in 20 years.

Adherence (MPR) was a composite measure assimilated from core medications that were present on an established questionnaire, the DMI-CF [7]. It is probable that each medication or treatment has a different weighting of importance both within and between patients. In turn this highlights the complexity of developing a valid adherence index measure for wider use. It is also likely that the composite measure used within this study could be reduced to contain fewer medications, although consensus is lacking as to what this might be.

The study has several limitations that are inherent to all studies examining adherence through cross-sectional design and that rely on current methods of measuring adherence. First, adherence is known to be a fluid measure, changing over time and by treatment component $[29,30]$. Second, measurement is complex, and although MPR is considered a more accurate measure of adherence than self-report, it has inherent limitations associated with changes in prescription and medication carryover. The former was partially accounted for within the study by accurate prescription data documented within the EHR system against which prescription collection could be aligned. For treatments such as pancreatic enzyme replacement therapy, in which patients self-titrate against differing snack and meal content, this is less useful; whilst average daily intake of pancreatic enzyme replacement therapy was obtained and documented, error is likely. Medication event monitoring system data, considered the "gold standard", can provide greater accuracy [31], but has inherent costs and its own bias if doses are removed but not consumed. These issues have led to the recognition that studies should incorporate more than one measure [7]. Our own results demonstrate concordance between both measures of adherence and have enabled patterns of objective measures of adherence to emerge that can inform future adherence interventions and provided predictive variables that can be further explored to aid evaluation.

The measure of lung function itself is also prone to error. We sought to minimise this through a standardised approach to measurement of pulmonary function within the clinic setting, in which trained physiotherapists undertook all measures in line with current guidance [20]. In healthier subjects, FEV1 is known to lack sensitivity in detecting early changes when in fact lung damage is present [32]. The threshold for variation to occur can therefore be different in early disease compared to moderate and late disease despite similar levels of poor adherence and this requires further study. In future research the more sensitive lung function clearance index could add further value and accuracy in determining smaller changes in lung function measurement [33]. 
The study also has a number of strengths. EHRs that contain data captured in "real time" enabled accurate extraction of all FEV1 data points for calculation of CoV FEV1 [17]. As a measure, CoV FEV1 provides an average of lung function dispersion values over time, making use of longitudinal data that can be incorporated into a single index. This is considered important in future studies, in a move away from "snapshot" values that could contribute to bias. Importantly, the final model was robust, meeting the validity criteria for ordinal regression and achieving a model of good fit.

\section{Conclusion}

$\mathrm{CoV}$ FEV1 is a significant predictor of adherence. This novel marker of adherence requires further evaluation across treatment regimens and duration of treatment.

\section{Acknowledgments}

Author contributions: H. White was involved in the conception, design, data analysis, interpretation, drafting/critical revision of the work and final approval. N. Shaw, S. Denman, K. Pollard and S. Wynne were involved in data acquisition, critical revision and final approval. D.G. Peckham was involved in the conception, design, data interpretation, critical revision and final approval.

\section{References}

1 Elborn JS, Bell SC, Madge SL, et al. Report of the European Respiratory Society/European Cystic Fibrosis Society task force on the care of adults with cystic fibrosis. Eur Respir J 2015; 47: 20-28.

2 Dodge JA, Lewis PA, Stanton M, et al. Cystic fibrosis mortality and survival in the UK: 1947-2003. Eur Respir J 2007; 29: 522-526.

3 Modi AC, Lim CS, Yu N, et al. A multi-method assessment of treatment adherence for children with cystic fibrosis. J Cyst Fibros 2006; 5: 177-185.

4 Daniels T, Goodacre L, Sutton C, et al. Accurate assessment of adherence. Self-report and clinician report vs electronic monitoring of nebulisers. Chest 2011; 140: 425-432.

5 Masterson TL, Wildman BG, Newberry BH, et al. Impact of age and gender on adherence to infection control guidelines and medical regimens in cystic fibrosis. Pediatr Pulmonol 2011; 46: 295-301.

6 Briesacher BA, Quittner AL, Salman L, et al. Adherence with tobramycin inhaled solution and health care utilization. BMC Pulm Med 2011; 11: 5.

7 Quittner AL, Modi AC, Lemanek KL, et al. Evidence-based assessment of adherence to medical treatments in pediatric psychology. J Pediatr Psychol 2008; 33: 916-936.

8 Eakin MN, Bilderback A, Boyle MP, et al. Longitudinal association between medication adherence and lung health in people with cystic fibrosis. J Cyst Fibros 2011; 10: 258-264.

9 Quittner A, Zhang J, Marynchenko M, et al. Pulmonary medication adherence and health-care use in cystic fibrosis. Chest 2014; 146: 142-151.

10 Gamble J, Stevenson M, McClean E, et al. The prevalence of nonadherence in difficult asthma. Am J Respir Crit Care Med 2009; 180: 817-822.

11 Gulini M, Prados C, Perez A, et al. Quality of life and adherence to nebulised antibiotic therapy using a new device in non-cystic fibrosis bronchiectasis. Enferm Clin 2012; 22: 148-153.

12 Vestbo J, Anderson JA, Calverley PMA, et al. Adherence to inhaled therapy, mortality and hospital admission in COPD. Thorax 2009; 64: 939-943.

13 Muntner P, Levitan EB, Joyce C, et al. Association between antihypertensive medication adherence and visit-to-visit variability of blood pressure. J Clin Hypertens 2013; 15: 112-117.

14 Hong K, Muntner P, Kronish I, et al. Medication adherence and visit-to-visit variability of systolic blood pressure in African Americans with chronic kidney disease in the AASK trial. J Hum Hypertens 2016; 30: 73-78.

15 Krakoff LR. Fluctuation: does blood pressure variability matter? Circulation 2012; 126: 525-527.

16 Hsiau M, Fernandez HE, Gjertson D, et al. Monitoring nonadherence and acute rejection with variation in blood immunosuppressant levels in pediatric renal transplantation. Transplantation 2011; 92: 918-922.

17 Morgan WJ, VanDevanter DR, Pasta DJ, et al. Forced expiratory volume in 1 second variability helps identify patients with cystic fibrosis at greater loss of lung function. J Pediatr 2016; 169: 116-121.

18 Rau JL. Determinants of patient adherence to an aerosol regimen. Respir Care 2005; 50: 1346-1356.

19 Peckham D, Etherington C, White $\mathrm{H}$, et al. The development and deployment of integrated electronic care records in a regional adult and paediatric cystic fibrosis unit. J Cystic Fibros 2014; 3: 681-686.

20 Miller MR, Hankinson J, Brusasco V, et al. Standardisation of spirometry. Eur Respir J 2005; 26: 319-338.

21 Smith NA, Seale JP, Ley P, et al. Better medication compliance is associated with improved control of childhood asthma. Monaldi Arch Chest Dis 1994; 49: 470-474.

22 Balfour-Lynn IM, Lees B, Hall P, et al. Multicenter randomized controlled trial of withdrawal of inhaled corticosteroids in cystic fibrosis. Am J Respir Crit Care Med 2006; 173: 1356-1362.

23 Arias Llorente RP, Bousono Garcia C, Diaz Martin JJ. Treatment compliance in children and adults with cystic fibrosis. J Cyst Fibros 2008; 7: 359-367.

24 Latchford G, Duff A, Quinn J, et al. Adherence to nebulised antibiotics in cystic fibrosis. Patient Educ Couns 2009; 75: 141-144.

25 George M, Rand-Giovanetti D, Eakin MN, et al. Perceptions of barriers and facilitators: self-management decisions by older adolescents and adults with CF. J Cyst Fibros 2010; 9: 425-432.

26 Bregnballe V, Schiotz PO, Boisen KA, et al. Barriers to adherence in adolescents and young adults with cystic fibrosis: a questionnaire study in young patients and their parents. Patient Prefer Adherence 2011; 5: 507-515.

27 Sawicki GS, Sellers DE, Robinson DM. High treatment burden in adults with cystic fibrosis: challenges to disease self-management. J Cyst Fibros 2009; 8: 91-96.

28 O'Donohoe R, Fullen BM. Adherence of subjects with cystic fibrosis to their home program: a systematic review. Respiratory Care 2014; 59: 1731-1746. 
29 Conway SP, Pond MN, Hamnett T, et al. Compliance with treatment in adult patients with cystic fibrosis. Thorax 1996; 51: 29-33.

30 Abbott J, Dodd M, Bilton D, et al. Treatment compliance in adults with cystic fibrosis. Thorax 1994; 49: 115-120.

31 Wildman MJ, Hoo ZH. Moving cystic fibrosis care from rescue to prevention by embedding adherence measurement in routine care. Paediatr Respir Rev 2014; 15: Suppl. 1, 16-18.

32 Brody AS, Klein JS, Molina PL, et al. High-resolution computed tomography in young patients with cystic fibrosis: distribution of abnormalities and correlation with pulmonary function tests. J Pediatr 2004; 145: 32-38.

33 Horsley AR, Gustafsson PM, Mcleod KA, et al. Lung clearance index is a sensitive, repeatable and practical measure of airways disease in adults with cystic fibrosis. Thorax 2008; 63: 135-140. 